\title{
ISOMORPHISMS OF PRIME GOLDIE SEMIPRINCIPAL LEFT IDEAL RINGS
}

\author{
KENNETH G. WOLFSON
}

(Communicated by Donald S. Passman)

\begin{abstract}
A prime (left) Goldie semiprincipal left ideal ring is the endomorphism ring $E(F, A)$ of a free module $A$, of finite rank, over a (left) Ore domain $F$. We examine the uniqueness of the module $(F, A)$ in the sense of determining necessary and sufficient conditions that every isomorphism of $E(F, A)$ is induced by a semilinear module isomorphism of $(F, A)$.
\end{abstract}

Introduction. Let $K$ be a prime (left) Goldie ring in which each finitely generated left ideal is principal. Then $K$ is isomorphic to the endomorphsm $\operatorname{ring} E(F, A)$ of a free module $A$, of finite rank, over a (left) Ore domain $F[3,6,8]$. We examine the uniqueness of this representation in the following sense: What are necessary and sufficient conditions such that, whenever $K \cong E(F, A) \cong E(G, B)$ (with $A, B$ free and finitely generated over domains $F$ and $G$ ), every isomorphism of $E(F, A)$ upon $E(G, B)$ is induced by a (semilinear) module isomorphism of $(F, A)$ upon $(G, B)$ ? We are able to show (Theorem 3.1) that this is precisely when all minimal right annihilators of $K$ are mutually isomorphic (as right $K$ modules), or equivalently when $K$ is isomorphic to $E(F, A)$ with $F$, a semiprincipal left ideal domain. A key point is showing that $K$ is a Baer ring (Lemma 2.3).

If "semilinear module isomorphism" is understood to include mappings between extensions of the underlying modules $(F, A)$ and $(G, B)$ then every isomorphism of $E(F, A)$ onto $E(G, B)$ is induced by a semilinear module isomorphism (Theorem $3.2)$.

1. Definitions and preliminaries. All rings have identity elements. The left module $A$ over the ring $F$ will be denoted $(F, A)$ and its endomorphism ring (operating on the right of $A$ ) will be denoted $E(F, A) .(A, E)$ will denote the right $E$ module $A$. The statement that $(F, A, E)$ is a bimodule indicates that $A$ is to be considered as a left $F$ module and a right $E$ module.

The arguments of Baer [1, Proposition 5, p. 176] with minor modifications can be used to prove

LEMMA 1.1. If there is an idempotent $e$ in $E=E(F, A)$ such that Ae is free and cyclic, then the bimodule $(F, A, E)$ is isomorphic to the bimodule (eEe,eE,E) in the following sense:

(1) $F$ and $e E e$ are isomorphic rings; $A$ and $e E$ are isomorphic additive groups, and there is a semilinear module isomorphism of the left modules $(F, A)$ and $(e E e, e E)$.

(2) There is an $E$ isomorphism of the right modules $(A, E)$ and $(e E, E)$.

Received by the editors October 13, 1987.

1980 Mathematics Subject Classification (1985 Revision). Primary 16A65; Secondary 16A04, $16 \mathrm{A34}$. 
LEMMA 1.2. Let $(F, A)$ possess a free cyclic summand, where $F$ is a domain, and let $E=E(F, A)$ be its endomorphism ring. If $e E$ and $f E$ are $E$-isomorphic for each pair of primitive idempotents $e, f$ in $E$, then every indecomposable summand of $A$ is free and cyclic.

ProOF. Let $S$ be an indecomposable summand of $A$, and $f$ an idempotent in $E$ such that $S=A f$. Let $e$ be an idempotent for which $A e$ is free and cyclic. Then $e$ and $f$ are primitive since $A e$ and $A f$ are indecomposable summands of $A$. By Lemma 1.1, there exists a bimodule isomorphism $\phi$ of $(F, A, E)$ onto $(e E e, e E, E)$. Hence $(A \sigma) \phi=(A \phi) \sigma=(e E) \sigma$, for each $\sigma \in E(F, A)$. If we let $\sigma=f$ then $S \phi=(A f) \phi=(e E) f=e E f$. Since $e E$ and $f E$ are $E$-isomorphic, it follows that $e E f$ is free and cyclic over $e E e$ [7, Lemma 2.3, p. 326]. Since $\phi$ is a (semilinear) module isomorphism of $(F, A)$ and $(e E e, e E), S$ must be free and cyclic over $F$.

2. Prime, Goldie, semi-pli rings. A ring $K$ is a pli ring (semi-pli ring), if each left ideal (finitely generated left ideal) is a principal left ideal.

A nonzero left module is uniform if any two nonzero submodules have nonzero intersection.

We shall say $(F, A) \in \mathscr{F}$, if $F$ is a domain, and $(F, A)$ is a finitely generated free module. If $(F, A) \in \mathscr{F}$, then $E(F, A)$ is prime. Results of Goldie [3], Robson [8] and Jategaonkar [6] (see also [4, Chapter 4]) imply that Ore.

(1) If $(F, A) \in \mathscr{F}$, then $E(F, A)$ is a (left) Goldie ring if, and only if, $F$ is (left)

(2) If $K$ is a prime Goldie semi-pli ring, there exists a module $(F, A) \in \mathscr{F}$ such that $K \cong E(F, A)$.

The proof of Lemma 4.11 of Chapter 4 of [4] shows that $A$ may be chosen as any minimal right annihilator of $K$. (We do not need the specific identification of $F$.) If $\phi$ is the isomorphism of $K$ onto $E(F, A)$ then for $k \in K, x k^{\phi}=x \cdot k$, for each $x \in A$.

We need the following slight generalization of a result of Jategaonkar [6, Proposition 2.11 , p. 51]. His arguments can be followed with minor modifications.

LEMMA 2.1. Let $(F, A) \in \mathscr{F}$, with $A$ free on $n$ generators. If $E(F, A)$ is a Goldie semi-pli ring, then for every set $J_{1}, J_{2}, \ldots, J_{n}$ of nonzero finitely generated left ideals of $F$, we have $J_{1} \oplus J_{2} \oplus \cdots \oplus J_{n}$ is isomorphic (as a left $F$-module) to $(F, A)$.

REMARK 1. If $E(F, A)$ satisfies the hypothesis of the lemma, each finitely generated left ideal of $F$ is projective. Hence each finitely generated submodule of $A$ is projective.

A ring in which each left annihilator ideal is a principal left ideal generated by an idempotent is called a Baer ring (the terminology is due to Kaplansky). The proof of the following is straightforward and will be omitted.

LEMMA 2.2. Let $K$ be a Baer ring, and $e$ an idempotent in $K$. Then the following are equivalent:

(1) $e$ is primitive.

(2) $e K$ is a minimal right annihilator.

(3) Ke is a minimal left annihilator. 
If $S$ is a submodule of $A, L(S)$ will denote the left ideal of $E(F, A)$ consisting of all $\sigma$ in $E(F, A)$ for which $A \sigma \subseteq S$. For $\tau$ in $E(F, A), \mathscr{L}(\tau)$ will consist of all $\rho$ in $E(F, A)$ for which $\rho \tau=0$, while $N(\tau)$ will be the submodule of $A$ consisting of all $a$ in $A$ for which $a \tau=0$.

LEMMA 2.3. If $K$ is a prime, Goldie, semi-pli ring, then $K$ is a Baer ring.

ProOF. $K \cong E(F, A)$ where $A$ is free of finite rank $n$, over an Ore domain $F$. If $J$ is a left annihilator ideal of $E=E(F, A)$, then $J=\mathscr{L}(\sigma), \sigma \in E[2$, Theorem 3.7, p. 208]. But $\mathscr{L}(\sigma)=L[N(\sigma)]$ follows from the relevant definitions. We shall show that $N(\sigma)$ is a summand of $A$, from which it will follow that $L[N(\sigma)]$ is generated by an idempotent. Since $A$ is finitely generated, so is $A \sigma$. By Remark 1, following Lemma $2.1, A \sigma$ is projective. Since $A \sigma \cong A / N(\sigma)$, it follows that $N(\sigma)$ is a summand of $A$. The fact that $L[N(\sigma)]$ is generated by an idempotent now follows as in the first part of the proof in [1, Proposition 1, p. 178].

\section{The main theorems.}

THEOREM 3.1. Let $K$ be a prime left Goldie semi-pli ring. The following statements are equivalent:

(1) If $K \cong E(F, A), K \cong E(G, B)$ with $(F, A),(G, B) \in \mathscr{F}$, then every isomorphism of $E(F, A)$ upon $E(G, B)$ is induced by a semilinear module isomorphism of $(F, A)$ upon $(G, B)$.

(2) All minimal right annihilators of $K$ are isomorphic (as right $K$ modules).

(3) All minimal left annihilators of $K$ are isomorphic (as left $K$ modules).

(4) All finitely generated uniform left ideals of $K$ are isomorphic (as left $K$ modules).

(5) There exists a semi-pli domain $F$, and a module $(F, A) \in \mathscr{F}$ such that $K \cong E(F, A)$.

PROOF. (1) $\Rightarrow(2)$ Let $J_{1}, J_{2}$ be minimal right annihilators of $K$, so that $J_{1}=e K$, $J_{2}=f K$ with $e, f$ primitive idempotents of $K$ (Lemmas 2.2, 2.3). By item (2) preceding Lemma 2.1, there is an isomorphism $\alpha$ of $K$ onto $E(F, e K)$ such that if $k \in K, x k^{\alpha}=x \cdot k$, for all $x \in e K$, and an isomorphism $\beta$ of $K$ onto $E(G, f K)$ such that if $k \in K, y k^{\beta}=y \cdot k$ for all $y \in f K$.

Clearly $\phi=\alpha^{-1} \beta$ is an isomorphism of $E(F, e K)$ onto $E(G, f K)$. For each $k \in K, k^{\alpha}=\sigma \in E(F, e K)$, so that

$$
\sigma^{\phi}=\left(k^{\alpha}\right)^{\alpha^{-1} \beta}=k^{\beta} \in E(G, f K) .
$$

By assumption $\phi$ is induced by a semilinear isomorphism $\rho$ of $(F, e K)$ upon $(G, f K)$. In particular,

$$
y \sigma^{\phi}=y\left(\rho^{-1} \sigma \rho\right), \quad \text { for each } y \in f K,
$$

or letting $y=x \rho$, for $x \in e K$, we have

$$
(x \rho) \sigma^{\phi}=x(\sigma \rho)=(x \sigma) \rho, \quad \text { for each } x \in e K, \quad \text { or } \quad(x \rho) k^{\beta}=\left(x k^{\alpha}\right) \rho .
$$

Hence

$$
(x \rho) \cdot k=(x \cdot k) \rho, \quad \text { for each } x \in e K,
$$

so that $\rho$ is a $K$-isomorphism of the right annihilators $e K$ and $f K$. 
$(2) \Rightarrow(5)$ By items (1) and (2) preceding Lemma $2.1, K \cong E(F, A)$ with $(F, A) \in$ $\mathscr{F}$ and $F$, a left Ore domain. Now let $J$ be a nonzero finitely generated left ideal of $F$. Since $F$ is an Ore domain, $J$ is an indecomposable $F$ module. By Lemma 2.1, there exists an $F$-module $Q$ such that $(F, J \oplus Q) \cong(F, A)$. Under this isomorphism $J$ maps onto an indecomposable summand of $A$, which is free and cyclic by Lemma 1.2. Hence $J$ must be a principal left ideal.

To prove $(5) \Rightarrow(1)$, Let $(F, A),(G, B) \in \mathscr{F}$ with $K \cong E(F, A) \cong E(G, B)$. Let $K \cong E\left(F_{0}, A_{0}\right)$ with $\left(F_{0}, A_{0}\right) \in \mathscr{F}$, and $F_{0}$ a semi-pli domain. Then of course $E\left(F_{0}, A_{0}\right) \cong E(F, A)$. By results of Jategaonkar [6, Theorem 1.6, p. 37, Proposition 1.8 , p. 40 , and Lemma 2.8, p. 49] this latter isomorphism is induced by a (semilinear) module isomorphism of $\left(F_{0}, A_{0}\right)$ onto $(F, A)$. In particular, $F$ is a semi-pli domain. Now we can apply the same argument to the isomorphism of $E(F, A)$ and $E(G, B)$ to conclude that it is induced by a semilinear module isomorphism of $(F, A)$ upon $(G, B)$.

$(2) \Leftrightarrow(3)$ If $e, f$ are idempotents in any $\operatorname{ring} K$, then $e K \cong f K$ if, and only if, $K e \cong K f[5$, Corollary, p. 51].

$(4) \Leftrightarrow(5)$ is a result of Robson [8, Theorem 5.3, p. 627].

REMARK 1. The restriction that $F$ and $G$ be domains is essential. Let $F$ be a pli domain, and $G=E\left(F, F^{(n)}\right), n>1$. Let $B=G^{(m)}, m>1$, and $A=F^{(m n)}$. Then $E(F, A)$ and $E(G, B)$ are isomorphic prime, pli rings, with $F, G$, nonisomorphic pli rings.

REMARK 2. Swan [9, Lemma 1, p. 57] gives an example of an $\left(F^{\prime}, A^{\prime}\right) \in \mathscr{F}$ for which $E\left(F^{\prime}, A^{\prime}\right)$ is a pli ring, but $F^{\prime}$ is not a pli domain.

Theorem 3.1 then implies that if $K=E\left(F^{\prime}, A^{\prime}\right)$ is the ring of Swan's example there exist $(F, A)$ and $(G, B) \in \mathscr{F}$, with $K \cong E(F, A), K \cong E(G, B)$ but with an isomorphism of $E(F, A)$ upon $E(G, B)$ which is not induced by a semilinear module isomorphism of $(F, A)$ upon $(G, B)$. In fact it is shown in [10] that there exist $(F, A),(G, B) \in \mathscr{F}$ with $K \cong E(F, A) \cong E(G, B)$ but $F \not F$, so that there is no (semilinear) module isomorphism of $(F, A)$ upon $(G, B)$. Nevertheless, even in this case, every isomorphism of $E(F, A)$ upon $E(G, B)$ is induced by a (semilinear) module isomorphism if we are willing to include mappings between extensions of the modules $(F, A)$ and $(G, B)$.

THEOREM 3.2. Let $K$ be a prime (left) Goldie semi-pli ring, such that $K \cong$ $E(F, A), K \cong E(G, B)$ with $(F, A),(G, B) \in \mathscr{F}$. Then, there exist modules $(\bar{F}, \bar{A})$, $(\bar{G}, \bar{B})$, which are extensions of $(F, A)$ and $(G, B)$ respectively, such that every isomorphism of $E(F, A)$ upon $E(G, B)$ is induced by a semilinear module isomorphism of $(\bar{F}, \bar{A})$ upon $(\bar{G}, \bar{B})$.

Proof. Assume that $(F, A),(G, B) \in \mathscr{F}$, and $E(F, A)$ and $E(G, B)$ are isomorphic Goldie semi-pli rings. Since $F$ and $G$ are Ore domains, they have quotient division rings $\bar{F}$ and $\bar{G}$. Let $\bar{A}=\bar{F} \otimes_{F} A$, and $\bar{B}=\bar{G} \otimes_{G} B$. Then $\bar{A}$ and $\bar{B}$ are (left) vector spaces over $\bar{F}$ and $\bar{G}$ respectively and with the usual identification, $(F, A)$ and $(G, B)$ are embedded in $(\bar{F}, \bar{A})$ and $(\bar{G}, \bar{B})$, and $E(F, A)$ and $E(G, B)$ are embedded as (left) orders in $E(\bar{F}, \bar{A})$ and $E(\bar{G}, \bar{B})$ respectively [6, Proposition 2.14 , p. 20]. If $\phi$ is an isomorphism of $E(F, A)$ upon $E(G, B)$, it has a unique extension to $\bar{\phi}$ an isomorphism of $E(\bar{F}, \bar{A})$ upon $E(\bar{G}, \bar{B})$. Hence there exists a one-one semilinear transformation $\rho$ of $(\bar{F}, \bar{A})$ onto $(\bar{G}, \bar{B})$ such that $\sigma^{\bar{\phi}}=\rho^{-1} \sigma \rho$ 
for each $\sigma \in E(\bar{F}, \bar{A})\left[1\right.$, Theorem 1, p. 183]. In particular $\sigma^{\phi}=\rho^{-1} \sigma \rho$ for each $\sigma \in E(F, A)$.

\section{REFERENCES}

1. R. Baer, Linear algebra and projective geometry, Academic Press, New York, 1952.

2. A. W. Goldie, Semiprime rings with maximum condition, Proc. London Math. Soc. 10 (1960), 201-220.

3. _ Non-commutative principal ideal rings, Arch. Math. 13 (1962), 214-221.

4. I. N. Herstein, Topics in ring theory, Univ. of Chicago Press, Chicago, Ill., 1969.

5. N. Jacobson, Structure of rings, Amer. Math. Soc. Colloq. Publ., vol. 37, Amer. Math. Soc., Providence, R. I., 1956.

6. A. V. Jategaonkar, Left principal ideal rings, Lecture Notes in Math., vol. 123, Springer-Verlag, Berlin and New York, 1970.

7. W. Liebert, Endomorphism rings of free modules over principal ideal domains, Duke Math. J. 41 (1974), 323-328.

8. J. C. Robson, Rings in which finitely generated right ideals are principal, Proc. London Math. Soc. 17 (1967), 617-628.

9. R. G. Swan, Projective modules over group rings and maximal orders, Ann. of Math. (2) 76 (1962), 55-61.

10. K. G. Wolfson, Isomorphisms of prime Goldie semi-principal left ideal rings. II, Canad. Math. Bull. (3) 31 (1988), 111-116.

Department of Mathematics, RUtGers University, NeW BRUNSWICK, NeW JeRSEY 08903 